\title{
Research on the joint inventory of repairable spare parts
}

\author{
Sheng Zhong, a, *, Shiming Yuan ${ }^{1, b}$, Wenjing Huang ${ }^{1, c}$ \\ 1Business School, Sichuan University, Chengdu 610065, China; \\ szhong666@163.com,ysmingscu@163.com,498368714@qq.com
}

Keywords: Pooling of spare parts; cost allocation; Spare parts repairation; value estimation; low-carbon

\begin{abstract}
Many enterprises which form the foundation of the society such as nuclear plant, manufacturing fields have confronted the difficulty of high cost in their capital management. In general, both the holding cost and shortage cost of spare parts owned by the enterprises is costly. Some literatures show that pooling of spare parts among multiple enterprises in the same region could reduce cost by scale economy and ensure the operating system security. In this essay, one high-cost but low-efficient spare part pooling management mode is studied. It is assumed that each player faces the Poisson Demand process and the stock point operates under a continuous-review policy. Moreover, in order to make the mode more close to reality and environmentally friendly, the condition in which the broken spare parts could be repaired, which refers to the reevaluation of the salvage value, has also been considered. And we also propose a cost allocation policy for the mode under this condition.
\end{abstract}

\section{Introduction}

Many enterprises which form the foundation of the society such as nuclear plant, manufacturing fields have confronted the difficulty of high cost in their capital management. The basic systems are automated, advanced and modernized, and relies heavily on equipment. To ensure the safety and efficiency of the system and to avoid the opportunity cost of the shutdown, the enterprise must reserve sufficient spare parts to ensure that the damaged parts can be replaced and repaired in time. However, the utilization of such spare parts is usually low, so that various types of spare parts will increase the management expenses and inventory costs, which will affect the economic benefits of enterprises.Normally, companies in the same region can jointly stock spare parts, it's an effective strategy to improve system availability. And Inventory pooling can bring economies of scale while reducing total costs.

The spare parts are usually very sophisticated and expensive, and the actual damaged spare parts won't be thrown away directly. This paper is based on the research on the joint inventory management of spare parts, and considered that the damaged spare parts can be repaired and reused. It is not only closer to the facts, but also meet the requirements of the green development of modern society. This paper will use the theory of cooperative game to solve these problems and construct a reasonable cost-sharing mechanism under the situationg that the spare parts are repairable. In practice, it is important for the enterprises to decide whether or not to build or participate in the spare parts pooling alliance, and to judge whether the method of cost allocation is reasonable.

\section{Literature review}

Spare parts have a great economic influence around the world, and there is a large number of related researches on it. The first relevant literature was published in 1966 by Feeney and Sherbrooke, indicating that in the spare parts stock model which considered in the paper, the quantity of replenishment of spare parts is subject to some stable probability distribution ${ }^{[1]}$. Slikker, Fransoo and Wouters ${ }^{[2]}$, Dror ${ }^{[3]}$, Chen and Zhang ${ }^{[4]}$, Kemahlioglu Ziya and Bartholdi ${ }^{[5]}$ have done some related research of the newsvendor model, the participants faced with stochastic market demand, and 
they can cooperate to decide order amount and manage their inventory together. Hartman and Dror assumed that the demand is stochastic, and regardlessed of the time of shortage, and establish a continuous model of inventory in a continuous observation state, and studied the approximate estimate $^{[6]}$. In contrast, in the model of this paper, it is assumed that the shortage cost per unit time is a determined value. From the point of the life-cycle of parts, Cui studies the optimal ordering time and order quantity of spare parts of high value and low flow rate ${ }^{[7]}$. The research direction of inventory pooling of spare parts is relatively new and the literature is relatively few. In 2007, Wong, van Oudheusden and Cattrysse first studied several enterprises in different regions cooperating to carry out inventory pooling under the condition of continuous observation, and put forward several ideas about cost allocation ${ }^{[8]}$. F Karsten, M Slikker and GJV Houtum considered several independent decision makers who stock expensive, low-demand spare parts for their high-tech machines ${ }^{[9]}$. S Benjaafar, WL Cooper, JS Kim studied inventory pooling in systems with symmetric costs where supply lead times are endogenously generated by a finite-capacity production system ${ }^{[10]}$. In $2010, \mathrm{~F}$ Karsten, M Slikker, GJV Houtum studied a situation where $\mathrm{n}$ independent companies separately stock spare parts of the same item for a technically advanced machine and they may reduce expected joint holding and downtime costs by pooling inventory ${ }^{[11]}$. In 2014, Frank Karsten and Rob J.I. Basten studied the inventory pooling of spare parts, and assumed that the inventory pooling management was decided by all participants together, and raised the topic of how to distribute the benefits and costs fairly ${ }^{[12]}$. In 2015, M Guajardo and M Rönnqvist considered an inventory pool of spare parts, subject to a service level constraint, where the members of the pool may have different target service levels, so that they represent different demand classes ${ }^{[13]}$.

The primary research on repairable spare parts is repairable spare parts multi-stage inventory theory, which is based on Sherbrooke's Metric model ${ }^{[14]}$ and Vari Metric model ${ }^{[15]}$. JPJV Kooten and $\mathrm{T}$ Tan considered a manufacturer of complex machines that offers service contracts to her customers, committing herself to repair failed spare parts throughout a fixed service period ${ }^{[16]}$. S Digiesi, G Mossa and S Rubino gived a contribution to the concept of sustainable management of repairable spare parts, by defining a repairable spare parts logistic model ${ }^{[17]}$.

In 2005, HW Wong presents a number of modeling and solution approaches for investigating how the use of pooling contributes to the goal of increased service performance and reduced cost in the context of repairable spare parts inventory management ${ }^{[18]}$. Hartanto Wong, Dirk Cattrysse and Dirk Van Oudheusden develop an analytical model to estimate several performance measures in a single-item, multi-company, repairable inventory system where complete pooling of stock is permitted among the companies. Compared to other research, this paper is different in that both non-zero lateral transshipment time and delayed lateral transshipments are considered in the model $^{[19]}$.

\section{Model}

3.1 (S-1, S) inventory model of repairable spare

\subsubsection{Assessment of damaged parts}

This article considers the large and expensive spare parts which can be regarded as fixed assets. And use the depreciation-straight line to make depreciation of the fixed assets.

Annual depreciation of assets $=\frac{\text { The original cost of the asset-Salvage value }}{\text { Service life }}$

Suppose the original cost of spare parts is $K$, the service life is $d$, the salvage value is $\alpha$, the annual depreciation of assets is $\beta$. Then $\beta=\frac{K-\alpha}{d}$. Suppose it has been used $r \quad(r<d)$ years since the spare parts broke down. If choose to repair spare parts, the spare parts can continue to use $(d-r)$ years, if choose not to repair spare parts, there will be no difference between the value of damaged spare parts and the value of spare parts that have expired the service year. The value is $\alpha$.

Suppose the maintenance costs of repairing the damaged spare parts is $T_{R}$.If the average annual cost of repairing spare parts is less than the annual depreciation expense of using the new spare parts, 
the spares will be chosen to repair. Otherwise, the spares will be chosen not to repair, which is:

When $\frac{T_{R}}{d-r}<\frac{K-\alpha}{d}$, spares are chosen to repair. When $\frac{T_{R}}{d-r}>\frac{K-\alpha}{d}$, spares are not chosen to repair.

Let $K_{R}$ be express the new cost of repairing broken-down spare parts, $K_{R}=\alpha+T_{R} \cdot K_{R}<K$, and prove as follows:

Because when spares are chosen to repair, $\frac{T_{R}}{d-r}<\frac{K-\alpha}{d}$, which is also $\frac{d}{d-r} T_{R}+\alpha<K$. And because of $\frac{d}{d-r} T_{R}>T_{R}$,it can be figured that $T_{R}+\alpha<K$, which means $K_{R}<K$.

\subsubsection{Model establishment and analysis}

Suppose a warehouse stores only one type of spare part, and the Initial stock is $S \in N_{0}$ ( $N_{0}=N \cup\{0\}$ ). The demand process for spare parts obeys the Poisson distribution with parameter $\lambda$ in unit time. When the stock is sufficient, the demand can be satisfied immediately. The whole process follows the FCFS (first come first serve) principle. The control of inventory adopt the continuous test strategy ( $S, S-1$ ), which means the warehouse will apply immediately to add one when use one. So the inventory level can be kept at $S$ from start to finish.

There will be an outward indent when out of stock. The time interval, which is from ordering to receiving, obeys independent identical distribution. In general, it is assumed that the mean is a unit time. When equipment parts are damaged, the damaged parts will be immediately sent to a repair shop for repair. Suppose that the repair shop has no capacity limit, and the time interval, which is from the begin to the finish of a part repair, is also subject to independent identical distribution whose mean is a unit time.

The $x(\lambda)$ is used to express the demand of inventory spares in one unit time, and $x(\lambda)$ obeys Poisson distribution with the expectation of $\lambda$. All $X \in N_{0}$ are satisfied: $P[X(\lambda)=x]=\frac{\lambda^{x}}{x !} e^{-\lambda}$

The out-of-stock quantity $B(S, \lambda)$ and the stock quantity $I(S, \lambda)$ have function relationship with the stock level $S$ and the demand rate $\lambda$. When the order quantity is greater than the basic inventory $S$, there will be a out-of-stock situation. Thus, the quantity of shortage will be $B(S, \lambda)=\max \{X(\lambda)-S, 0\}$.

Similarly, $I(S, \lambda)=\max \{S-X(\lambda), 0\}$. According to (1), the probability distribution and expectation of the out-of-stock quantity and the inventory quantity can be figured out. The probability distributions of the out-of-stock quantity $B(S, \lambda)$ are as follows:

$$
P[B(S, \lambda)=x]=\left\{\begin{array}{l}
\sum_{y=0}^{S} P[X(\lambda)=y] \\
P[X(\lambda)=x+S]
\end{array} \quad \text { if } \quad x \in N=0(2)\right.
$$

And the expectation of Out-of-stock quantity is:

$$
\begin{aligned}
E B(S, \lambda) & =\sum_{x=S+1}^{\infty}(x-S) P[X(\lambda)=x] \\
& =\sum_{x=0}^{\infty} x * P[X(\lambda)=x]-\sum_{x=0}^{\infty} S * P[X(\lambda)=x]+\sum_{x=0}^{S}(S-x) P[X(\lambda)=x] \\
& =\lambda-S+\sum_{x=0}^{S}(S-x) P[X(\lambda)=x]
\end{aligned}
$$

Similarly, the expectation of inventory quantity can be figured out:

$$
E I(S, \lambda)=\sum_{x=0}^{S}(S-x) P[X(\lambda)=x]=E B(S, \lambda)-\lambda+S(4
$$

The expectation of damage to machine parts in one unit time is $\lambda$. Suppose the repair rate of damaged spare parts is a (The repair rate is the ratio between repairable spares which passed the assessment in 4.1 and the total quantity of damaged spares), $0<a<1$. The expectation of new spare parts replaced in one unit time is $(1-a) * \lambda$, and the expectation of replaced spare parts which has been repaired is $a * \lambda$. The cost of a single new spare part is $K$, and the cost of a single repaired spare part is $K_{R}$, which is: The long term average cost of spare parts in one unit time 
$=(1-a) * \lambda * K+a * \lambda * K_{R}$.

It is assumed that the cost of a single spare part in one unit time is $b, b>0$. The inventory costs include the use cost of the warehouse, the insurance cost of spare parts and the opportunity cost of the funds which can bear interest and be occupied by the fixed assets. The prices are different between repaired spare parts and new spare parts, and the corresponding inventory costs are also different. It is assumed that the inventory cost of each new spare part in a unit time is $h, h>0$; and the inventory cost of each repair spare parts per unit time is $h_{R}, h_{R}>0$. Because of $K_{R}<K$, there will be $h_{R}<h$. Suppose that the number of spare parts in the warehouse accounts for $\beta$ of the total number of spare parts, the long-term average inventory cost per unit time will be: $h *(1-\beta) * E I(S, \lambda)+h_{R} * \beta * E I(S, \lambda)$ 。

Therefore, in the case of repairable spare parts, the long-term average cost per unit time is:

$C_{R}(S, \lambda)=(1-a) * \lambda * K+a * \lambda * K_{R}+h *(1-\beta) * E I(S, \lambda)+h_{R} * \beta * E I(S, \lambda)+b * E B(S, \lambda)$

\section{Cooperative Games Based on Cost Allocation}

\subsection{Correlative Theories}

Definition 1: For a game that has $n$ participants, $N=\{1,2, \ldots, n\}$, any $M \subseteq N$ will be known as a coalition belongs to $N$. In exceptional case, the $M$ can be valued $M=\varnothing$ or $M=N$.And when $M=N$, the $M$ is known as a big coalition ${ }^{[20]}$.

If the income of participants in the coalition can be freely allocated, and the income of any participant changes 1 unit, its utility also changes 1 unit. This situation is called (transformable utility, TU) . This article only discusses situation in transformable utility.

Definition 2: Given the participants of cost allocation game as $N=\{1,2, \ldots, n\}$, then the $c(M)$ will be a real-valued function which is defined on all subsets(The coalition) of $N$. And it can also express the cost function of coalition $M$ which satisfied $c(\varnothing)=0 ; c(N) \leq \sum_{i \in N} c(\{i\})$. The $c(M)$ will be known as a characteristic function and the game will be denoted by $G=[N, c]{ }^{[20]}$

In the cooperative games based on cost allocation, the characteristic function $c(M)$ of coalition $M$ represents the opportunity cost of independent activities in coalition $M$. In this article, $c(M)$ represents coalition $M$ which means the long-term average total cost per unit time for the $M$ participants to manage spare parts by inventory pooling.

In a cooperative game $G=[N, c]$ with $n$ participants, if it is given that n-dimensional vector $x=\left(X_{1}, X_{2}, \ldots, X_{n}\right)$ satisfies two conditions:

Condition 1: $x_{i} \leq c(\{i\}), \quad i=1,2, \ldots, n$

Condition 2: $\sum_{i=1}^{n} x_{i}=c(N)(7)$

The n-dimensional vector $x$ will be one kind of cost allocations of the cooperative game $G=[N, c]$, and $X_{i}$ will be the value that the participants $i$ have allocated.

The allocation with stability will satisfy all individuals and the coalition. This allocation will make any participant dispel motivation for splitting the coalition. Then we can define the core of cooperative game as follows:

Definition3 : Given $G=[N, c]$ as a cooperative game with $n$ participants, if there is one kind of cost allocation $x_{x}=\left(X_{1}, X_{2}, \ldots, X_{n}\right)$ make all the $S \subseteq N$ satisfy $\sum_{\mathrm{i} \in M} X_{i} \leq c(M)$, this set made up of allocation $X$ will be known as the core of game $G$ and be recorded as $C(N, c)$. The stable cost allocation of big coalition is the core of the cooperative games based on cost allocation, 
which means

$$
C(N, c)=\left\{x \in R^{N} \mid \sum_{i \in N} X_{i}=c(N) \text { 且 } \sum_{\mathrm{i} \in \mathrm{M}} X_{i} \leq c(M), M \neq N\right\} \text { 。 }
$$

Even if the sum of the costs of any combination of participants in big coalition is less than the sum of costs of any small coalition or single one of them, the core of the cooperative game may still be empty set.

Practically, participants are usually only interested in a coalition of strictly better outcomes. And there will be a consideration for reinforcing the core.

Definition 4: It is given that $G=[N, c]$ is a cooperative game with $n$ participants, $C_{0}(N, c)$ is reinforced core of the game. If the $C_{0}(N, c)$ satisfies the following condition:

$$
C_{0}(N, c)=\left\{x \in R^{N} \mid \sum_{i \in N} X_{i}=c(N) \text { 且 } \sum_{i \in \|,} X_{i}<c(M), M \neq N\right\} \text { 。 }
$$

The reinforced core ensures that the total cost of any member of the big coalition is better than the total cost of the small one they organized. The core can prevent the emergence of small coalition and maintain the stability of big coalition.

\subsection{Analysis of Inventory pooling of Repairable Spare Parts}

It is considered that there are several enterprises carrying out jointly inventory management of the same spare part. The demand of each enterprise (which is participant) follows a stable Poisson distribution, and the demand process of each participant is of independent identical distribution. Suppose that expectation of replenishment time of each participant is equal, in general, the expectation of replenishment time is assumed as 1 . Participants are generally firms operating in the same industry environment, assuming that inventory costs and cost outs are consistent.

Use $\varphi$ to express the jointly inventory management under the condition of repairable spare parts, $\varphi=\left(N,\left(\lambda_{i}\right)_{i \in N}, K, K_{R}, a, h, h_{R}, \beta, b\right), N$ represents the nonempty set of participants, $\lambda_{i}>0$ is the spare demand rate of participants $i$ in the set $N, K$ is the cost of a single new spare part, $K_{R}$ is the cost of repairing a single spare part, $a(0<a<1)$ is the repair rate of damaged spare parts, $h(h>0)$ is the inventory cost of every new spare part per unit time, $h_{R}\left(h_{R}>0\right)$ is the inventory cost of each repaired spare part per unit time, $\beta$ is the ratio of the number of repaired spare parts to the total quantity of spare parts in the inventory, and $b(b>0)$ is the out-of-stock cost of each spare part in one unit time.

In the management of spare parts jointly inventory of any coalition, the inventory level suitable for the coalition will be set up according to the sum of the participants' needs, and the demand of the participants for the jointly inventory spare parts follows the First-Come-First-Served principle. The sum of Poisson distributions of several independent identical distribution is still Poisson distribution, Therefore, the demand rate of the joint inventory level still obeys Poisson distribution, and the joint inventory demand rate of the coalition $M$ is $\lambda_{M}=\sum_{i \in, M} \lambda_{i}$.

\subsection{Cooperative Advantages of Inventory Pooling}

It has been introduced in the Introduction that the spare parts jointly inventory management is suitable for the enterprises with high spare parts prices and low utilization rate. In general, enterprises have a certain inventory level before the management of joint inventory. Due to the particularity of spare parts, enterprises can not choose spare parts for sale or reproduction. Therefore, the initial inventory level of the spare parts jointly inventory management is the sum of the original inventory of each participant, and the jointly inventory management of the spare parts can reduce the cost and benefit the participants. In this case, the jointly inventory management of the spare parts strictly reduces the expected shortage quantity( (3) ), strictly reduces the expected inventory quantity((4)), and reduces the expected cost strictly.

Proposition 1: Consider a set of cooperative game with $n$ participants as $N$, and for any 
participant $i \in N$, the demand rate is $\lambda_{i}$ and the inventory spare parts level $S_{i}$. It is assumed that $\sum_{i \in N} S_{i}>0,|N| \geq 2$

$$
\begin{aligned}
& \sum_{i \in N} E B\left(S_{i}, \lambda_{i}\right)>E B\left(\sum_{i \in N} S_{i}, \sum_{i \in N} \lambda_{i}\right) ; \\
& \sum_{i \in N} E I\left(S_{i}, \lambda_{i}\right)>E I\left(\sum_{i \in N} S_{i}, \sum_{i \in N} \lambda_{i}\right) ; \\
& \sum_{i \in N} c\left(S_{i}, \lambda_{i}\right)>c\left(\sum_{i \in N} S_{i}, \sum_{i \in N} \lambda_{i}\right) \circ
\end{aligned}
$$

Proof procedure: It is considered that at any point in time, $\left(y_{i}\right)_{i \in N}$ is the spare parts requirement of each participant and it obeys Poisson distribution of expectation $\lambda_{i} \cdot\left(y_{i}\right)_{i \in N}$ is also a value of the Poisson distribution at a certain time point. If the spare parts demand reaches $y=\left(y_{i}\right)_{i \in N}$, under the circumstance of not carrying out the spare parts jointly inventory management, the sum of out-of-stock quantity in each enterprise will be: $\sum_{i \in N} \max \left\{y_{i}-S_{i}, 0\right\}$; and under the circumstance of carrying out the spare parts jointly inventory management the sum of out-of-stock quantity of each participant will be: $\max \left\{\sum_{i \in N} y_{i}-\sum_{i \in N} S_{i}, 0\right\}$. And the value of $\sum_{i \in N} \max \left\{y_{i}-S_{i}, 0\right\}$ must be greater than or equal to the value of $\max \left\{\sum_{i \in N} y_{i}-\sum_{i \in N} S_{i}, 0\right\}$. For two different participants $i, j \in N$, if the condition $y_{i}-S_{i}<0$ and $y_{j}-S_{j}>0$ can be satisfied, the value of $\sum_{i \in N} \max \left\{y_{i}-S_{i}, 0\right\}$ will be strictly greater than the value of $\max \left\{\sum_{i \in N} y_{i}-\sum_{i \in N} S_{i}, 0\right\}$. The intuitive understanding of (8) can be explained as that, a participant can use the inventories of other participants to reduce the incidence of shortage after the jointly inventory management of spare parts. Similarly, (9) and (10) can be proved according to (4) and (5).

If the basic inventory level of the jointly inventory is maintained at the sum of the basic inventory levels of the participants when they work on their own, the jointly inventory management can indeed reduce costs. In fact, with the management of spare parts joint inventory, if the big coalition reset the optimal basic inventory level, the cost will still be reduced. The basic level of reset inventory is lower than the sum of the basic inventory levels of the participants when they work on their own, which may lead to an increase in the expected shortage compared with no cooperation. But at the same time, the inventory on hand will be significantly reduced, and the reduction of inventory cost will balance out the increase of the out-of-stock cost. Common management and lower inventory level will make the cooperation more low-cost and high-efficiency.

\section{Cost Allocation System of Inventory Pooling of Repairable Spare Parts}

The long-term average cost $C_{R}(S, \lambda)$ in a unit time is affected by the basic inventory quantity $S$ of spare parts joint inventory. The choice of any $S \in N_{0}$ has a corresponding $C_{R}(S, \lambda)$. It is assumed that if an arbitrary coalition $M$ selects the optimal basic inventory quantity $S_{0}$, the optimal long-term average cost of the coalition $M$ will be $C_{R}\left(S_{0}, \lambda_{M}\right)$ per unit time, which means $C_{R}\left(S_{0}, \lambda_{M}\right)=\min _{S \in N_{0}} C_{R}\left(S, \lambda_{M}\right)$.

It is of great significance for the stability of the coalition to ensure the fair and rational allocation of the long-term average cost with jointly inventory management.

Under the condition of repairable spare parts, the long-term average cost per unit time can be divided into 3 parts: The cost of using new spare parts and repaired spare parts in a unit time is denoted by $C_{R 1}(S, \lambda)$. The inventory cost of new spare parts and repaired spare parts in a unit time is denoted by $C_{R 2}(S, \lambda)$. And the shortage cost in a unit time is denoted by $C_{R 3}(S, \lambda)$.

Namely,

$$
\begin{aligned}
C_{R}(S, \lambda) & =C_{R 1}(S, \lambda)+C_{R 2}(S, \lambda)+C_{R 3}(S, \lambda) \\
& =(1-a) * \lambda * K+a * \lambda * K_{R}+h *(1-\beta) * E I(S, \lambda)+h_{R} * \beta * E I(S, \lambda)+b * E B(S, \lambda)
\end{aligned}
$$


Firstly, the sum of the cost of using the new spare parts and the repaired spare parts for the big coalitionis allocated. One kind of method of allocation, which supposes that the total cost of the spare parts used by the enterprise was undertaken by themselves 'own, can be carried out. If an enterprise $i$ used $m$ new spares and $n$ repaired spares per unit time. The line of the cost of repairing $n$ spare parts goes to $K_{R 1}, K_{R 2}, \ldots, K_{R n}$, and the cost should be undertaken by the participant $i$ per unit time will be $C_{(R 1, i)}\left(S, \lambda_{i}\right)=m * K+K_{R 1}+K_{R 2}+\ldots+K_{R n}$.

The principle of multi-use and multi-undertake, which is simple and fair, can be used for the allocation of cost and inventory, which means that anyone who uses more inventory spares should undertake more cost. And the allocation of the cost is according to demand ratio of the inventory spares. For instance, with the spare parts repairable jointly inventory management $\varphi=\left(N,\left(\lambda_{i}\right)_{i \in N}, K, K_{R}, a, h, h_{R}, \beta, b\right)$, the total demand rate of the big coalition is $\lambda_{N}=\sum_{i \in N} \lambda_{i}$, the inventory cost that any participant enterprise $i(i \in N)$ should undertake is $C_{(R 2, i)}\left(S, \lambda_{i}\right)=C_{R 2}\left(S, \lambda_{i}\right) * \frac{\lambda_{i}}{\lambda_{N}}$, and the shortage cost is $C_{(R 3, i)}\left(S, \lambda_{i}\right)=C_{R 3}\left(S, \lambda_{i}\right) * \frac{\lambda_{i}}{\lambda_{N}}$.

Therefore, the cost undertaken by any participant $i(i \in N)$ in the jointly inventory management with repairable spare parts is:

$$
\begin{aligned}
& C_{(R, i)}\left(S, \lambda_{i}\right) \\
& =C_{(R 1, i)}\left(S, \lambda_{i}\right)+C_{(R 2, i)}\left(S, \lambda_{i}\right)+C_{(R 3, i)}\left(S, \lambda_{i}\right) \\
& =m * K+K_{R 1}+K_{R 2}+\ldots+K_{R n}+C_{R 2}\left(S, \lambda_{i}\right) * \frac{\lambda_{i}}{\lambda_{N}}+C_{R 3}\left(S, \lambda_{i}\right) * \frac{\lambda_{i}}{\lambda_{N}} \\
& =m * K+K_{R 1}+K_{R 2}+\ldots+K_{R n}+\left\{h *(1-\beta) * E I\left(S, \lambda_{i}\right)+h_{R} * \beta * E I\left(S, \lambda_{i}\right)\right\} * \frac{\lambda_{i}}{\lambda_{N}}+b * E B\left(S, \lambda_{i}\right) * \frac{\lambda_{i}}{\lambda_{N}}
\end{aligned}
$$

\section{Conclusions}

Based on the analysis of existing joint management of spare parts, this paper adds the consideration that the spare parts can be repaired and reused, which makes the model more close to the actual situation, meets the development requirements of green and low carbon. And the advantages of jointly inventory management to the enterprise are also be analyzed. The reduction of costs and the steady cooperative relationship make the jointly inventory management of repairable spare parts practicable in application, especially the enterprises with high inventory and shortage cost who urgently needs a reasonable way of cooperation to reduce costs.

\section{References}

[1] Feeney GJ, Sherbrooke CC, The S-1, S Inventory Policy Under Compound Poisson Demand. INFORMS: 1966; p 391-411.

[2] Slikker M, Fransoo J, Wouters M. Joint ordering in multiple news-vendor problems: a game-theoretical approach [J]. 2001,

[3] Dror M, Guardiola LA, Meca A, et al. Dynamic realization games in newsvendor inventory centralization [J]. International Journal of Game Theory, 2008, 37(1):139-153.

[4] Chen X, Zhang J. A Stochastic Programming Duality Approach to Inventory Centralization Games [J]. Operations Research, 2009, 57(4):840-851.

[5] Kemahl, Lu-Ziya E, Bartholdi JJ. Centralizing Inventory in Supply Chains by Using Shapley Value to Allocate the Profits [J]. Manufacturing \& Service Operations Management, 2011, 13(2):146-162.

[6] Hartman BC, Dror M. Cost allocation in continuous-review inventory models [J]. Naval Research Logistics, 1996, 43(4):549-561. 
[7] 崔南方, 丁留明. 基于零件寿命函数的慢速流动备件库存模型 [J]. 系统工程, 2006, 24(9):24-31.

[8] Wong H, Oudheusden DV, Cattrysse D. Cost allocation in spare parts inventory pooling [J]. Transportation Research Part E Logistics \& Transportation Review, 2007, 43(4):370-386.

[9] Karsten F, Slikker M, Houtum GJV. Inventory pooling games for expensive, low-demand spare parts [J]. Naval Research Logistics, 2012, 59(5):311-324.

[10] Benjaafar S, Cooper WL, Kim JS. On the Benefits of Pooling in Production-Inventory Systems [J]. Management Science, 2005, 51(4):548-565.

[11] Karsten F, Slikker M, Houtum GJV. Resource Pooling and Cost Allocation Among Independent Service Providers [J]. Operations Research, 2015, 63(2):págs. 476-488.

[12] Karsten F, Basten RJI. Pooling of spare parts between multiple users: How to share the benefits? [J]. European Journal of Operational Research, 2014, 233(1):94-104.

[13] Guajardo M, Rönnqvist M. Cost allocation in inventory pools of spare parts with service-differentiated demand classes [J]. International Journal of Production Research, 2015, 53(1):220-237.

[14] Sherbrooke CC. Metric: A Multi-Echelon Technique for Recoverable Item Control [J]. Operations Research, 1966, 16(1):122-141.

[15] In VARI-METRIC: Improved approximation for multi-indenture, multi-echelon availability models, Operations Research, 1986.

[16] Kooten JPJV, Tan T. The Final Order Problem for Repairable Spare Parts under Condemnation [J]. Journal of the Operational Research Society, 2009, 60(10):1449-1461.

[17] Digiesi S, Mossa G, Rubino S. Sustainable Order Quantity of Repairable Spare Parts [J]. IFAC Proceedings Volumes, 2012, 45(31):181-186.

[18] Wong HW. Pooling of repairable spare parts: A study on inventory policies [J]. 4OR, 2005, 3(3):253-256.

[19] Wong H, Cattrysse D, Oudheusden DV. Stocking decisions for repairable spare parts pooling in a multi-hub system [J]. International Journal of Production Economics, 2005, s 93-94(1):309-317.

[20] 汪贤裕, 肖玉明, 博栾论及其应用. 科学出版社: 2008; p 82-84. 